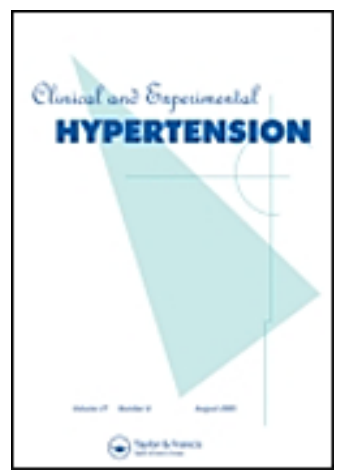

\title{
Effects of blackcurrant extract on arterial functions in older adults: A randomized, double-blind, placebo-controlled, crossover trial
}

\begin{tabular}{|r|l|}
\hline Journal: & Clinical and Experimental Hypertension \\
\hline Manuscript ID & LCEH-2020-0069 \\
\hline Manuscript Type: & Original Papers \\
\hline Author: & 09-Mar-2020 \\
\hline Complete List of Authors: & $\begin{array}{l}\text { Okamoto, Takanobu; Nippon Sport Science University, Department of } \\
\text { Exercise Physiology } \\
\text { Hashimoto, Yuto; Nippon Sport Science University, Graduate School of } \\
\text { Health and Sport Science } \\
\text { Kobayashi, Ryota; Teikyo University of Science, Center for Fundamental } \\
\text { Education } \\
\text { Nakazato, Koichi; Nippon Sport Science University, Department of } \\
\text { Exercise Physiology } \\
\text { Willems, Mark; University of Chichester, Institute of Sport }\end{array}$ \\
\hline Keywords: & $\begin{array}{l}\text { Anthocyanins, Pulse wave velocity, Augmentation index, Vascular } \\
\text { function, Cardiovascular risk factors }\end{array}$ \\
\hline
\end{tabular}

\section{SCHOLARONEm Manuscripts}




\section{Clinical and Experimental Hypertension}

2

3

4

5

6

7

8

9

10 Takanobu Okamoto, $\mathrm{PhD}$, Yuto Hashimoto, MS, Koichi Nakazato, $\mathrm{PhD}$

11 Department of Exercise Physiology, Nippon Sport Science University, Tokyo, Japan

12 Ryota Kobayashi, PhD

13 Center for Fundamental Education, Teikyo University of Science, Tokyo, Japan

14 Mark Elisabeth Theodorus Willems, PhD

15 Institute of Sport, University of Chichester, Chichester, United Kingdom

16 Corresponding author

17 Takanobu Okamoto

18 ORCID ID: 0000-0003-1978-2455

19 7-1-1, Fukasawa, Setagaya-ku, Tokyo, 158-8508, Japan

20 Tel: +81-3-5706-0966

21 Fax: +81-3-5706-0966

22 e-mail: tokamoto@nittai.ac.jp

Original Papers

\section{Effects of blackcurrant extract on arterial functions in older adults: A} $\mathrm{PhD}$, Mark Elisabeth Theodorus Willems, $\mathrm{PhD}$ 
ABSTRACT

24 Purpose: Blackcurrant extract mainly contains anthocyanins. Several reports suggest that

25 anthocyanins have beneficial effect for cardiovascular functions. The aim of this study was to

26 examine the effect of 7-day intake of New Zealand blackcurrant (NZBC) extract on arterial

27 functions, e.g. arterial stiffness, and serum lipids.

28 Methods: A randomized, double-blind, placebo-controlled, crossover design study with a

29 washout period of 28 days was conducted. Fourteen older adults participated in this study (age

$3073.3 \pm 1.7$ years). Participants took either a 7-day course of placebo or two capsules of NZBC

31 extract (each $300 \mathrm{mg}$ capsule contains 35\% blackcurrant extract). Participants took one of the

32 two trials first and then took the other after a washout period. Carotid-femoral pulse-wave

33 velocity, an index of central arterial stiffness, and central blood pressure were measured at

34 baseline and again at the end of the 7-day study period.

35 Results: Compared to baseline, carotid-femoral pulse-wave velocity $(P=0.03)$ and central

36 blood pressure $(\mathrm{P}=0.02)$ decreased significantly after the 7-day study period with NZBC

37 intake. In addition, carotid-femoral pulse-wave velocity $(\mathrm{P}=0.04)$ and central blood pressure

$38(\mathrm{P}=0.001)$ in the NZBC intake trial decreased significantly more than in the placebo intake

39 trial. No effects were observed on serum lipids.

40 Conclusion: These results suggest that short-term NZBC intake reduces central arterial 41 stiffness and central blood pressure in older adults. Therefore, anthocyanin-rich blackcurrants 
42 might be beneficial for maintaining or improving cardiovascular health as an alternative to 43 pharmaceutical medications.

44

45 Key words: Anthocyanins, Pulse wave velocity, Augmentation index, Vascular function, 46 Cardiovascular risk factors

48 Abbreviations

49 AIx, augmentation index; BP, blood pressure; cfPWV, carotid-femoral pulse-wave velocity;

50 CVD, cardiovascular diseases; DBP, diastolic blood pressure; faPWV, femoral-ankle pulse-

51 wave velocity; FG, fasting glucose; HDL, high-density lipoprotein cholesterol; LDL, low-

52 density lipoprotein cholesterol; MBP, mean blood pressure; NZBC, New Zealand blackcurrant;

53 PP, pulse pressure, SBP, systolic blood pressure; TG, triglycerides 


\section{INTRODUCTION}

Mortality due to cardiovascular disease is a serious global problem (1), and many

strategies are needed to prevent cardiovascular disease. An increase in central arterial stiffness

57 as assessed by carotid-femoral pulse wave velocity (cfPWV) is a powerful predictor of future

58 cardiovascular events, such as myocardial infarction or stroke, and all-cause mortality (2-4). In

59 addition, central aortic blood pressure (BP) has greater prognostic capability than brachial BP,

60 and is more strongly related to vascular hypertrophy, the extent of atherosclerosis, and other

61 cardiovascular events (3-6). Therefore, minimizing increases in central arterial stiffness and

62 central BP are important for the prevention of cardiovascular disease (CVD).

63 In previous randomized trials, it has been reported that consumption of polyphenols

64 from foods or extracts significantly improved vascular health. In fact, polyphenol-rich foods

65 such as berries, chocolate, or cocoa reduced brachial BP or central arterial stiffness and

66 improved vascular endothelial function $(7,8)$. Prospective studies investigating the association

67 between flavonoid consumption and myocardial infarction risk in young and middle-aged

68 adults showed that high intake of anthocyanins present in blueberries and strawberries is

69 associated with a decreased risk of myocardial infarction (9). Anthocyanins are among the

70 polyphenols that are part of the flavonoid family. Anthocyanins have potent antioxidant

71 capacity and/or a powerful vasodilator effect by producing nitric oxide (10-13). It is known

72 that short-term anthocyanin consumption significantly reduces arterial stiffness and improves 
73 vascular endothelial function compared to placebo in young and middle-aged adults (14-17).

74 In addition, according to a meta-analysis by Fairlie-Jones et al.(18) acute intake of anthocyanin

75 significantly improved arterial stiffness in healthy young adults. On the other hand,

76 blackcurrants include specific anthocyanins, consisting primarily of delphinidin-3-rutinoside,

77 delphinidin-3-glucoside, cyanidin-3-rutinoside, and cyanidin-3-glucoside, which have

78 numerous health benefits $(19,20)$. Thus, intake of anthocyanin-rich blackcurrants may have a

79 number of beneficial effects on cardiovascular health. However, to the best of our knowledge,

80 the effects of short-term blackcurrant consumption on central arterial stiffness and central BP

81 in older adults are unknown. More research is needed to determine various strategies to achieve

82 cardioprotective effects in older adults.

83 The aim of this study was to examine the effect of 7-day intake of New Zealand

84 blackcurrant (NZBC) extract on arterial functions, such as arterial stiffness and serum lipids.

85 We hypothesized that short-term NZBC intake reduces central arterial stiffness and blood 86 pressure in older adults. 


\section{METHODS}

88

89

90

$91 \quad$ Participants

92

93

94

obtained from all participants after providing detailed explanations of the potential risks of the

104 study. A health history questionnaire was completed to confirm health states. Table 1 shows

105 the physical characteristics of the participants. The study protocol was approved by the ethics

Fourteen volunteers (female: 8 , male: 6 ) more than 65 years old were recruited from the area near Nippon Sport Science University in Japan. Figure 1 shows the flow diagram of enrollment and trial assignment of the participants. Inclusion criteria were: 1. non-smoking; 2. not obese (body mass index $\leq 30 \mathrm{~kg} / \mathrm{m}^{2}$ ); 3 . $\geq 65$ years of age; 4. no history of cardiovascular disease or diabetes; 5. no diseases or disorders affecting their daily life; 6 . not taking medication that compromises the cardiovascular system, including antihyperlipidemic, antihypertensive, or antihyperglycemic medications; 7. not taking supplementation; 8. not taking habitual exercise; 9. postmenopausal women; and 10. not taking hormone replacement therapy. The inclusion criteria of each participant were confirmed by the authors during screening before the experiment. The number of participants who met the inclusion criteria and who were willing to participate in this study totaled 14 (female: 8, male: 6). Written, informed consent was 
106 committee of Nippon Sport Science University (Japan) (018-H087).

108 Schedule

109

Participants visited the laboratory four times at the same time in the morning (8:00

110 am). Figure 2 shows the experimental schedule. Before arrival, participants were instructed to

111 avoid vigorous exercise for 48 hours, alcohol for 24 hours and caffeine-containing products on

112 the day of testing and be fasted for at least 10-14 hours overnight.

At every visit, arterial stiffness and BP were measured and blood samples collected

114 from the ulnar vein of the nondominant arm of each participant.

$116 \quad$ NZBC and placebo intake

117 Participants ingested $2 \times 300 \mathrm{mg}$ capsules (total $210 \mathrm{mg}$ of anthocyanins) of

118 concentrated NZBC extract or a visually identical placebo for 7 days. Each $300 \mathrm{mg}$ NZBC

119 capsule contained $105 \mathrm{mg}$ of anthocyanins, consisting of 35-50\% delphinidin-3-rutinoside, 5-

$12020 \%$ delphinidin-3-glucoside, $30-45 \%$ cyanidin-3-rutinoside, and $3-10 \%$ cyanidin-3-

121 glucoside (CurraNZ®, Health Currancy Ltd., Surrey, UK). Each placebo capsule contained

$122300 \mathrm{mg}$ microcrystalline cellulose._Two capsules were consumed with breakfast for the first 6

123 days. On the final morning of the supplementation period, participants were instructed to take

124 the supplement or placebo without breakfast, with the last intake about $2 \mathrm{~h}$ before an 
125 experimental visit. The two experimental conditions (NZBC extract and placebo) were 126 separated by a 4-week washout period (21). Intake of beverages such as green tea and/or dietary 127 supplements that included polyphenols were prohibited during the study. No restrictions were 128 placed on food intake, but all participants were instructed to maintain their normal diet and 129 lifestyle throughout the study period.

130

131 Body composition

132 The body composition of the participants was determined by bioelectric impedance 133 using a body-composition analyzer (InBody770, Biospace Co Ltd, Seoul, South Korea).

\section{Arterial stiffness}

136 cfPWV and femoral-ankle PWV (faPWV), which reflect aortic and leg arterial 137 stiffness, respectively, were measured. Carotid and femoral artery-pressure waveforms were 138 obtained for 30 seconds using arterial applanation tonometry incorporating form PWV/ABI 139 micropiezoresistive transducers (Omron-Colin Co Ltd, Tokyo, Japan), which comprise 15 140 aligned pressure-sensitive elements that can identify carotid and femoral pulse traces, arranged 141 side by side and attached to the left common carotid and femoral arteries, respectively. A 142 vascular testing device (form PWV/ABI; Omron-Colin) was used to measure simultaneously 143 electrocardiograms, bilateral brachial and ankle BP, and carotid-arterial and femoral-arterial 
144 pulse waves (PWs). cfPWV and faPWV were calculated by dividing the distance between the

145 two arterial recording sites by the transit time, which is determined based on the time delay

146 between the proximal and distal "foot" waveforms. faPWV is calculated based on the transit

147 time between the femoral artery site and the ankle site. Next, time delays were obtained from

148 between the right brachial and posttibial arteries, the carotid and femoral arteries (Tcf), and the

149 femoral and posttibial arteries. A nonelastic tape measure was then used to make duplicate

150 random zero-length measurements over the body surface in order to determine the path length

151 from the carotid to the femoral artery (Dcf). cfPWV was calculated using the following

152 equation:

153

$154 \quad \mathrm{cfPWV}=\mathrm{Dcf} / \mathrm{Tcf}$

155

156

The investigator who performed the PWV measurements was blinded to the condition

157 assignment of the participants.

158

159 Central and brachial BPs and the augmentation index (AIx)

Arterial pulse waveforms of the left radial artery for estimating central BP were

161 measured non-invasively by an automated tonometric system (HEM-9000AI, Omron

162 Healthcare Co., Ltd.). Central BP and the AIx were measured as previously described $(22,23)$. 
163 Brachial BP was measured with an oscillometric manometer, and radial pulse waveforms were

164 recorded non-invasively using an applanation tonometer. Signals of the radial arterial pressure

165 wave were low-pass-filtered, first at a cut-off frequency of $105 \mathrm{~Hz}$ to remove high-frequency

166 noise and then at $25 \mathrm{~Hz}$ to extract pressure waveforms. Inflection points or peaks that

167 corresponded to first (early) and second (late) systolic BP were automatically identified using

168 the fourth derivative wave as the second and third zero crossing points, respectively. Late SBP

169 in the radial artery was used as an estimate of the central SBP and was calculated using the

170 following equation:

171 Late $\mathrm{SBP}=(\mathrm{P} 2 / \mathrm{PP}) \times(\mathrm{SBP}-\mathrm{DBP})+\mathrm{DBP}$

172 In addition, the radial AIx was calculated using the following equation:

$173 \quad \operatorname{AIx}(\%)=(\mathrm{P} 2 / \mathrm{PP}) \times 100$

174 where $\mathrm{P} 2$ is the pressure difference between the peak systolic pressure and an early inflection

175 point that indicates the beginning upstroke of the reflected pressure wave, and PP is the pulse 176 pressure.

177 The AIx was adjusted for a heart rate of 75 bpm (AIx@75).

178 In addition, mean brachial BP (MBP) and brachial pulse pressure (PP) were calculated

179 using the following equations:

$180 \mathrm{MBP}=[\mathrm{DBP}+(\mathrm{SBP}-\mathrm{DBP}) / 3]$.

$181 \quad \mathrm{PP}=\mathrm{SBP}-\mathrm{DBP}$ 
182

183 Serum lipids profile and glucose

184

Whole blood was drawn into serum separator tubes, allowed to clot and centrifuged at

185 4,000 RCF for 15 minutes. Triglyceride (TG), high-density lipoprotein cholesterol (HDL-C),

186 low-density lipoprotein cholesterol (LDL-C) and fasting glucose (FG) in blood samples were

187 measured using standard enzymatic techniques. The intra- and interassay coefficients of

188 variance was less than $5 \%$.

189

190 Statistics

191 All data are expressed as means \pm standard error. Statistical analyses were performed

192 using statistical software (SPSS ver. 24; SPSS, Inc., Chicago, IL, USA). The assumption of a

193 normal distribution for all data was verified using the Kolmogorov-Smirnov test, and all data

194 were normally distributed. Data were analyzed using two-way analysis of variance (trials $\times$

195 periods) with repeated measures. When the main effect or interaction was significant, the paired

196 t-test was used to identify significant differences among the mean values. Statistical

197 significance was set at $\mathrm{P}<0.05$. In addition, The interpretation of $\mathrm{p}$ as $0.05>\mathrm{p} \leq 0.1$ was

198 according to guidelines by Curran-Everett and Benos (24). 
RESULTS

200

Figure 3 shows changes in cfPWV before and after both NZBC and placebo. The

201 cfPWV at baseline did not differ significantly between NZBC and placebo. cfPWV decreased

202 significantly after NZBC intake compared with baseline values $(\mathrm{P}=0.03)$. In contrast, the

203 cfPWV did not differ significantly between before and after placebo intake. Significant

204 differences in cfPWV were observed between the NZBC and placebo after intervention $(\mathrm{P}=$ $2050.04)$.

206 Figure 4 shows changes in central BP before and after both NZBC and placebo. The 207 central BP at baseline did not differ significantly between NZBC and placebo. Central BP 208 decreased significantly after NZBC intake compared with baseline values $(\mathrm{P}=0.02)$. In 209 contrast, the central BP did not differ significantly before and after placebo intake. Significant 210 differences in central BP were observed between the NZBC and placebo after intervention (P $211=0.001)$

Table 2 shows changes in brachial SBP, DBP, MBP, PP, AIx, and faPWV before and 213 after NZBC and placebo. The brachial SBP, DBP, MBP, PP, AIx, and faPWV at baseline did 214 not differ significantly between NZBC and placebo. Brachial SBP $(\mathrm{P}=0.03), \mathrm{DBP}(\mathrm{P}=0.02)$, $215 \operatorname{MBP}(\mathrm{P}=0.01)$, and AIx $(\mathrm{P}=0.03)$ decreased significantly after NZBC intake compared with 216 baseline values. PP had a tendency to decrease after NZBC intake compared with baseline 217 values $(\mathrm{P}=0.09)$. In contrast, brachial SBP, DBP, MBP, PP and AIx did not differ significantly 
218 between before and after placebo intake. Significant differences in brachial SBP $(P=0.001)$, 219 MBP $(\mathrm{P}=0.01), \mathrm{PP}(\mathrm{P}=0.01)$, and AIx $(\mathrm{P}=0.01)$ were observed between the NZBC and 220 placebo trials after intervention $(\mathrm{P}=0.001)$. There was a trend for $\mathrm{DBP}$ to be lower with NZBC 221 trial compared with placebo trial after intervention $(p=0.07)$. On the other hand, faPWV did 222 not differ significantly between before and after both NZBC and placebo. In addition, no 223 significant differences in faPWV were observed between the NZBC and placebo trials after 224 intervention.

225 Table 3 shows changes in serum lipids profile before and after both NZBC and placebo.

226 No significant differences were seen between NZBC and placebo in serum concentrations of 227 TG, HDL-C, LDL-C, or FG at baseline. No significant changes were observed between the two 228 trials in serum concentrations of TG, HDL-C, LDL-C, or FG after both NZBC and placebo. 


\section{DISCUSSION}

230

The key finding of this study was that 7 days intake of anthocyanin-rich NZBC in

231 older adults decreased cfPWV and cSBP with no changes in the placebo condition. These

232 results suggest that short-term intake of NZBC would decrease central arterial stiffness and BP

233 in older adults.

234

An increase in central arterial stiffness and/or central BP has been shown to be

235 independently associated with future cardiovascular events, such as myocardial infarction or

236 stroke, and all-cause mortality $(25,26)$. Therefore, intake of NZBC may be an important type

237 of non-pharmacological therapy to enhance cardiovascular health in older adults.

238 The present findings show for the first time that anthocyanin-rich foods such as NZBC

239 reduce arterial stiffness in older adults. Anthocyanins are flavonoids, all of which are phenolic

240 compounds (i.e., polyphenols). Recent studies have shown that ingestion of anthocyanins-rich

241 foods, including black currants, blueberries, grapes, and purple potatoes, have the beneficial

242 effects of reducing arterial stiffness in both young and middle-aged adults, and growing

243 evidence highlights that specific flavonoids from plant bioactive compounds present in fruits

244 improve vascular function $(27,28)$. Some previous studies that examined the associations

245 between anthocyanin intake and arterial stiffness focused predominantly on young and middle-

246 aged participants. In our recent study, it was shown that intake of polyphenol-rich cocoa

247 reduced cfPWV in postmenopausal women (8). In addition, previous studies showed an 
248 association between polyphenol-rich food intake and decreased aortic stiffness in healthy adults,

249 independent of age $(3,4,29,30)$. Consistent with previous studies $(8,31)$, the present results 250 suggest that intake of polyphenol-rich foods, i.e. anthocyanin-rich blackcurrant, improves 251 central arterial stiffness. Thus, the present findings expand on previous research on the effects 252 of polyphenol-rich foods on arterial stiffness in older adults.

253 In addition to the improvements in central arterial stiffness, 7-day intake of NZBC 254 extract was shown, for the first time, to reduce central BP by average of $10 \mathrm{mmHg}$, which 255 appears to be a better predictor of cardiovascular events than brachial BP (3-6). Wang et al. 256 (32) observed central BP reductions of $6 \mathrm{mmHg}$ that reduced cardiovascular mortality by 257 approximately $25 \%$. Thus, the present findings showed that NZBC intake reduced central BP, 258 suggesting the effectiveness of anthocyanin-rich foods for reducing central BP.

259 In the previous meta-analyses of randomized controlled trials, consumption of 260 flavonoid-rich foods (e.g., green tea, soy protein isolates, and cocoa or chocolate) was shown 261 to reduce peripheral (brachial) BP (33). Consistent with a previous study, the present findings 262 showed that intake of NZBC reduced brachial BP. Moreover, NZBC intake reduced the AIx. 263 The central aortic pressure wave consists of a forward traveling wave generated by left 264 ventricular ejection, followed by a later-arriving reflected wave from the periphery (34). In 265 addition, as arterial stiffness increases, central BP increases can occur due to increased forward 266 and reflected wave amplitudes and earlier return of the reflected wave to the proximal aorta. 
267 Reduction in the AIx is associated with increased peripheral vasodilation (35), caused by

268 decreased wave reflection at medium-sized muscular arteries (36). Therefore, the decreased

269 AIx in the present study may have been caused by peripheral vasodilation. Moreover, the

270 measurements of arterial stiffness obtained from non-invasive pressure waveforms suggested

271 that decreased aortic stiffness is associated with reduced AIx (37). Thus, the present findings

272 suggest that the changes in central arterial stiffness and BP after NZBC intake were primarily

273 the result of changes in arterial distension. Aortic stiffness affects both early systolic cardiac

274 load, through elevation of the forward pressure wave, and late systolic cardiac load, due to

275 earlier return of the reflected pressure wave (38-42). Thus, the present findings suggested that

276 the effect of NZBC intake was mediated by reductions in both early systolic and late systolic

277 pressures, suggesting decreased aortic stiffness and arteriolar tone, respectively. Therefore,

278 since decreased wave reflection decreases LV afterload and myocardial oxygen demand (43),

279 NZBC intake appears to be effective for reducing cardiovascular risk in older adults.

280 In the present study, there were no differences in serum lipids and glucose after intake

281 of NZBC or placebo. Alvarez-Suarez et al. (44) reported that one-month intake of anthocyanin-

282 rich strawberry improved the lipid profile by significantly reducing TC, LDL-C, and TG levels

283 compared to baseline levels, while no changes were seen in HDL cholesterol. In this respect,

284 the present results differ from that of previous study. One possible reason for this difference

285 may be differences in the subjects and intake duration among studies. Moreover, since TC, 
286 LDL-C, and TG in most participants of the present study were within the standard ranges, their 287 values might not have differed from baseline values. In addition, Tsang et al. (28) reported that 288 intake of an anthocyanin-rich potato did not change glucose. This result is consistent with our 289 findings. Thus, the present findings showed that NZBC intake did not change serum lipid 290 profile and glucose in older adults.

291 In individuals at risk, medical and pharmacologic interventions can decrease 292 cardiovascular mortality, but such interventions are costly and may have adverse effects 293 (45,46). In contrast, in epidemiological studies, higher anthocyanin intake was associated with 294 decreased arterial stiffness and BP $(47,48)$. Increased arterial stiffness or BP is a major CVD 295 risk factor (49), which, when decreased significantly, reduces the risk of CVD and death in 296 various populations (50). Various fruits, vegetables, and beverages that are commonly 297 consumed in the human diet can be rich in anthocyanins (51-53) Therefore, the present findings 298 emphasize the importance of incorporating more anthocyanin-rich foods, including fruits and 299 vegetables and berries such as NZBC, which promote cardiovascular health without side effects. This study has some limitations. First, the short time frame of the study might have 301 prevented observation of the benefits of longer-term consumption on arterial stiffness and BP. 302 Second, vascular endothelial function, antioxidant capabilities, and inflammatory markers were 303 not measured. Third, the results in this study population of healthy older adults might not be 304 generalizable to CVD patients. Finally, there was no information on anthocyanin-derived 
305 metabolites with 7-day NZBC intake. Nevertheless, to the best of our knowledge, the present

306 data provide the first evidence that short-term intake of NZBC may contribute to reductions in

307 central arterial stiffness and BP in older adults. Further long-term, randomized, interventional

308 studies are needed to establish the role of NZBC in supporting cardiovascular health in older 309 adults.

310

\section{Conclusion}

312 In conclusion, the findings of the present study indicate that short-term NZBC intake

313 reduces central arterial stiffness and BP in older adults. These results suggest that habitual

314 NZBC intake may be an effective way to prevent CVD. Therefore, anthocyanin-rich

315 blackcurrants might be beneficial for maintaining or improving cardiovascular health as an

316 alternative to pharmaceutical medications.

\section{Acknowledgement}

320 with the experiments.

\section{$322 \quad$ Funding}

There are no funding sources for the present study. 
324

325 Compliance with ethical standards

326 Conflict of interest

327 The authors have no conflict of interest to declare.

328 Statement of human and animal rights

329 This study was approved by the Ethics Committee of Nippon Sport Science University.

330 Informed consent

331 Informed consent was obtained from all participants. 


\section{REFERENCES}

333 1. Mortality GBD, Causes of Death C. Global, regional, and national age-sex specific allcause and cause-specific mortality for 240 causes of death, 1990-2013: a systematic analysis for the Global Burden of Disease Study 2013. Lancet. 2015;385(9963):11771. DOI:10.1016/S0140-6736(14)61682-2 PMCID:PMC4340604.

2. Scuteri A, Tesauro M, Appolloni S, Preziosi F, Brancati AM, Volpe M. Arterial 338 stiffness as an independent predictor of longitudinal changes in cognitive function in the older individual. J Hypertens. 2007;25(5):1035-40.

341 3. Vlachopoulos C, Aznaouridis K, O'Rourke MF, Safar ME, Baou K, Stefanadis C. Prediction of cardiovascular events and all-cause mortality with central haemodynamics: a systematic review and meta-analysis. Eur Heart J. 2010;31(15):1865-71. DOI:10.1093/eurheartj/ehq024

345 4. Vlachopoulos C, Aznaouridis K, Stefanadis C. Prediction of cardiovascular events and 346 all-cause mortality with arterial stiffness: a systematic review and meta-analysis. J Am 347 Coll Cardiol. 2010;55(13):1318-27. DOI:10.1016/j.jacc.2009.10.061

348 5. Cheng HM, Chuang SY, Sung SH, Yu WC, Pearson A, Lakatta EG, Pan WH, Chen $349 \mathrm{CH}$. Derivation and validation of diagnostic thresholds for central blood pressure measurements based on long-term cardiovascular risks. J Am Coll Cardiol. 
351 2013;62(19):1780-7. DOI:10.1016/j.jacc.2013.06.029 PMCID:PMC3884552.

352 6. Safar ME, Levy BI. Studies on arterial stiffness and wave reflections in hypertension. 353 Am J Hypertens. 2015;28(1):1-6. DOI:10.1093/ajh/hpu155

3547 Hooper L, Kay C, Abdelhamid A, Kroon PA, Cohn JS, Rimm EB, Cassidy A. Effects 355 of chocolate, cocoa, and flavan-3-ols on cardiovascular health: a systematic review and 356 meta-analysis of randomized trials. Am J Clin Nutr. 2012;95(3):740-51. 357 DOI:10.3945/ajcn.111.023457

358 8. Okamoto T, Kobayashi R, Natsume M, Nakazato K. Habitual cocoa intake reduces 359 arterial stiffness in postmenopausal women regardless of intake frequency: a randomized parallel-group study. Clin Interv Aging. 2016;11:1645-1652. DOI:10.2147/CIA.S118152 PMCID:PMC5115689.

362 9. Cassidy A, Mukamal KJ, Liu L, Franz M, Eliassen AH, Rimm EB. High anthocyanin 363

366 10. Edirisinghe I, Banaszewski K, Cappozzo J, McCarthy D, Burton-Freeman BM. Effect of black currant anthocyanins on the activation of endothelial nitric oxide synthase 368 369 intake is associated with a reduced risk of myocardial infarction in young and middleaged women. Circulation. 2013;127(2):188-96.

\section{DOI:10.1161/CIRCULATIONAHA.112.122408 PMCID:PMC3762447.} (eNOS) in vitro in human endothelial cells. J Agric Food Chem. 2011;59(16):8616-24. DOI:10.1021/jf201116y 
370 11. Edirisinghe I, Banaszewski K, Cappozzo J, Sandhya K, Ellis CL, Tadapaneni R,

371

372

374 12. Wallace TC. Anthocyanins in cardiovascular disease. Adv Nutr. 2011;2(1):1-7.

DOI:10.3945/an.110.000042 PMCID:PMC3042791.

376 13. Zafra-Stone S, Yasmin T, Bagchi M, Chatterjee A, Vinson JA, Bagchi D. Berry 377 anthocyanins as novel antioxidants in human health and disease prevention. Mol Nutr 378

379 14. Keane KM, George TW, Constantinou CL, Brown MA, Clifford T, Howatson G. 380

383 15. Rodriguez-Mateos A, Feliciano RP, Boeres A, Weber T, Dos Santos CN, Ventura MR, 384 385 386

387 16. Rodriguez-Mateos A, Rendeiro C, Bergillos-Meca T, Tabatabaee S, George TW, Heiss 388
Effects of Montmorency tart cherry (Prunus Cerasus L.) consumption on vascular function in men with early hypertension. Am J Clin Nutr. 2016;103(6):1531-9. DOI:10.3945/ajcn.115.123869 function: A double-blind, randomized, controlled, dose-response, crossover study. Mol Nutr Food Res. 2016;60(10):2130-2140. DOI:10.1002/mnfr.201600250

C, Spencer JP. Intake and time dependence of blueberry flavonoid-induced
Heiss C. Cranberry (poly)phenol metabolites correlate with improvements in vascular 
389 improvements in vascular function: a randomized, controlled, double-blind, crossover 390 intervention study with mechanistic insights into biological activity. Am J Clin Nutr. 391 2013;98(5):1179-91. DOI:10.3945/ajcn.113.066639

392 17. Zhu Y, Xia M, Yang Y, Liu F, Li Z, Hao Y, Mi M, Jin T, Ling W. Purified anthocyanin 393 supplementation improves endothelial function via NO-cGMP activation in 394 395 hypercholesterolemic individuals. Clin Chem. 2011;57(11):1524-33. DOI:10.1373/clinchem.2011.167361

396 18. Fairlie-Jones L, Davison K, Fromentin E, Hill AM. The Effect of Anthocyanin-Rich 397 398 399 DOI:10.3390/nu9080908 PMCID:PMC5579701.

400 19. Gavrilova V, Kajdzanoska M, Gjamovski V, Stefova M. Separation, characterization 401 and quantification of phenolic compounds in blueberries and red and black currants by 402 HPLC-DAD-ESI-MSn. J Agric Food Chem. 2011;59(8):4009-18. DOI:10.1021/jf104565y

404 20. Gopalan A, Reuben SC, Ahmed S, Darvesh AS, Hohmann J, Bishayee A. The health 405 benefits of blackcurrants. Food Funct. 2012;3(8):795-809. DOI:10.1039/c2fo30058c

406 21. Jin Y, Alimbetov D, George T, Gordon MH, Lovegrove JA. A randomised trial to 407 investigate the effects of acute consumption of a blackcurrant juice drink on markers of 
408

409

410

411

412

413

414

415

416

417 24. Curran-Everett D, Benos DJ. Guidelines for reporting statistics in journals published 418

425 26. Mitchell GF, Hwang SJ, Vasan RS, Larson MG, Pencina MJ, Hamburg NM, Vita JA, 426

vascular reactivity and bioavailability of anthocyanins in human subjects. Eur J Clin Nutr. 2011;65(7):849-56. DOI:10.1038/ejen.2011.55

22. Kumagai H, Zempo-Miyaki A, Yoshikawa T, Tsujimoto T, Tanaka K, Maeda S. Lifestyle modification increases serum testosterone level and decrease central blood pressure in overweight and obese men. Endocr J. 2015;62(5):423-30. DOI:10.1507/endocrj.EJ14-0555

23. Tanaka S, Sugiura T, Yamashita S, Dohi Y, Kimura G, Ohte N. Differential response of central blood pressure to isometric and isotonic exercises. Sci Rep. 2014;4:5439. DOI:10.1038/srep05439 PMCID:PMC4069636.

by the American Physiological Society. Am J Physiol Regul Integr Comp Physiol. 2004;287(2):R247-9. DOI:10.1152/ajpregu.00346.2004

25. Karras A, Haymann JP, Bozec E, Metzger M, Jacquot C, Maruani G, Houillier P, Froissart M, Stengel B, Guardiola P, Laurent S, Boutouyrie P, Briet M, Nephro Test Study G. Large artery stiffening and remodeling are independently associated with allcause mortality and cardiovascular events in chronic kidney disease. Hypertension. 2012;60(6):1451-7. DOI:10.1161/HYPERTENSIONAHA.112.197210

Levy D, Benjamin EJ. Arterial stiffness and cardiovascular events: the Framingham 
Study.

Circulation.

2010;121(4):505-11.

DOI:10.1161/CIRCULATIONAHA.109.886655 PMCID:PMC2836717.

429

27. Rodriguez-Mateos $\mathrm{A}$

A, Heiss C, Borges

G, Crozier

A. Berry (poly)phenols and 430 cardiovascula health. $\mathrm{J}$

Agric

Food

Chem.

2014;62(18):3842-51.

431

DOI:10.1021/jf403757g

432

28. Tsang C, Smail NF, Almoosawi S, McDougall GJM, Al-Dujaili EAS. Antioxidant Rich 433

Potato Improves Arterial Stiffness in Healthy Adults. Plant Foods Hum Nutr.

434 2018;73(3):203-208. DOI:10.1007/s11130-018-0673-2 PMCID:PMC6096904.

435

29. Grassi D, Desideri G, Necozione S, di Giosia P, Barnabei R, Allegaert L, Bernaert H, 436

Ferri C. Cocoa consumption dose-dependently improves flow-mediated dilation and 437 arterial stiffness decreasing blood pressure in healthy individuals. J Hypertens 438 2015;33(2):294-303. DOI:10.1097/HJH.0000000000000412

30. Heiss C, Sansone R, Karimi H, Krabbe M, Schuler D, Rodriguez-Mateos A, Kraemer 440 T, Cortese-Krott MM, Kuhnle GG, Spencer JP, Schroeter H, Merx MW, Kelm M, 441 Flaviola Consortium EUtFP. Impact of cocoa flavanol intake on age-dependent 442 vascular stiffness in healthy men: a randomized, controlled, double-masked trial. Age (Dordr). 2015;37(3):9794. DOI:10.1007/s11357-015-9794-9 PMCID:PMC4444618.

444 31. Vlachopoulos CV, Alexopoulos NA, Aznaouridis KA, Ioakeimidis NC, Dima IA, 445 Dagre A, Vasiliadou C, Stefanadi EC, Stefanadis CI. Relation of habitual cocoa 
446 consumption to aortic stiffness and wave reflections, and to central hemodynamics in

healthy individuals. Am J Cardiol.

2007;99(10):1473-5.

32. Wang KL, Cheng HM, Chuang SY, Spurgeon HA, Ting CT, Lakatta EG, Yin FC, Chou

$\mathrm{P}$, Chen $\mathrm{CH}$. Central or peripheral systolic or pulse pressure: which best relates to target

organs and future mortality? J Hypertens. 2009;27(3):461-7. PMCID:PMC3178100.

33. Hooper L, Kroon PA, Rimm EB, Cohn JS, Harvey I, Le Cornu KA, Ryder JJ, Hall WL, Cassidy A. Flavonoids, flavonoid-rich foods, and cardiovascular risk: a meta-analysis of randomized controlled trials. Am J Clin Nutr. 2008;88(1):38-50. DOI:10.1093/ajcn/88.1.38

34. O'Rourke MF, Staessen JA, Vlachopoulos C, Duprez D, Plante GE. Clinical 457 applications of arterial stiffness; definitions and reference values. Am J Hypertens. 2002;15(5):426-44. 459

35. Mahmud A, Feely J. Beta-blockers reduce aortic stiffness in hypertension but nebivolol, 460 not atenolol, reduces wave reflection. Am J Hypertens. 2008;21(6):663-7. 461 DOI:10.1038/ajh.2008.156

462 36. Wilkinson IB, MacCallum H, Flint L, Cockcroft JR, Newby DE, Webb DJ. The 463 influence of heart rate on augmentation index and central arterial pressure in humans. J Physiol. 2000;525 Pt 1:263-70. PMCID:PMC2269933. 
465 37. Nichols WW. Clinical measurement of arterial stiffness obtained from noninvasive pressure waveforms. Am J Hypertens. 2005;18(1 Pt 2):3S-10S. DOI:10.1016/j.amjhyper.2004.10.009

38. Borlaug BA, Melenovsky V, Redfield MM, Kessler K, Chang HJ, Abraham TP, Kass 469 DA. Impact of arterial load and loading sequence on left ventricular tissue velocities in humans. J Am Coll Cardiol. 2007;50(16):1570-7. DOI:10.1016/j.jacc.2007.07.032

39. Chirinos JA, Segers P, Raina A, Saif H, Swillens A, Gupta AK, Townsend R, Emmi 472 AG, Jr., Kirkpatrick JN, Keane MG, Ferrari VA, Wiegers SE, St John Sutton MG. Arterial pulsatile hemodynamic load induced by isometric exercise strongly predicts left ventricular mass in hypertension. Am J Physiol Heart Circ Physiol. 475 2010;298(2):H320-30. DOI:10.1152/ajpheart.00334.2009

40. Chirinos JA, Segers P, Rietzschel ER, De Buyzere ML, Raja MW, Claessens T, De 477 Bacquer D, St John Sutton M, Gillebert TC, Asklepios I. Early and late systolic wall 478 479 480 stress differentially relate to myocardial contraction and relaxation in middle-aged adults: the Asklepios study. Hypertension. 2013;61(2):296-303. DOI:10.1161/HYPERTENSIONAHA.111.00530

481 41. Laurent S, Boutouyrie P. Recent advances in arterial stiffness and wave reflection in 482 human hypertension. Hypertension. 2007;49(6):1202-6. 
484 42. Ooi H, Chung W, Biolo A. Arterial stiffness and vascular load in heart failure. Congest $485 \quad$ Heart Fail. 2008;14(1):31-6.

486 43. O'Rourke MF, Hashimoto J. Mechanical factors in arterial aging: a clinical perspective. 487 J Am Coll Cardiol. 2007;50(1):1-13. DOI:10.1016/j.jacc.2006.12.050

488 44. Alvarez-Suarez JM, Giampieri F, Tulipani S, Casoli T, Di Stefano G, Gonzalez489 Paramas AM, Santos-Buelga C, Busco F, Quiles JL, Cordero MD, Bompadre S, 490 Mezzetti B, Battino M. One-month strawberry-rich anthocyanin supplementation 491 ameliorates cardiovascular risk, oxidative stress markers and platelet activation in 492 humans. J Nutr Biochem. 2014;25(3):289-94. DOI:10.1016/j.jnutbio.2013.11.002

493 45. Abbott AL. Medical (nonsurgical) intervention alone is now best for prevention of 494 stroke associated with asymptomatic severe carotid stenosis: results of a systematic 495 review and analysis.

Stroke. 2009;40(10):e573-83.

46. O'Keefe JH, Carter MD, Lavie CJ. Primary and secondary prevention of cardiovascular 498 diseases: a practical evidence-based approach. Mayo Clin Proc. 2009;84(8):741-57. 499 DOI:10.1016/S0025-6196(11)60525-9 PMCID:PMC2719528.

500 47. Cassidy A, O'Reilly EJ, Kay C, Sampson L, Franz M, Forman JP, Curhan G, Rimm 501 EB. Habitual intake of flavonoid subclasses and incident hypertension in adults. Am J 502 Clin Nutr. 2011;93(2):338-47. DOI:10.3945/ajcn.110.006783 PMCID:PMC3021426. 
503 48. Jennings A, Welch AA, Fairweather-Tait SJ, Kay C, Minihane AM, Chowienczyk P, 504 Jiang B, Cecelja M, Spector T, Macgregor A, Cassidy A. Higher anthocyanin intake is 505 associated with lower arterial stiffness and central blood pressure in women. Am J Clin 506 Nutr. 2012;96(4):781-8. DOI:10.3945/ajen.112.042036

507 49. Lewington S, Clarke R, Qizilbash N, Peto R, Collins R, Prospective Studies C. Age508 specific relevance of usual blood pressure to vascular mortality: a meta-analysis of 509 individual data for one million adults in 61 prospective studies. Lancet. 510 2002;360(9349):1903-13.

511 50. Ettehad D, Emdin CA, Kiran A, Anderson SG, Callender T, Emberson J, Chalmers J, 512 Rodgers A, Rahimi K. Blood pressure lowering for prevention of cardiovascular 513 disease and death: a systematic review and meta-analysis. Lancet. 514 2016;387(10022):957-967. DOI:10.1016/S0140-6736(15)01225-8

515 51. Giampieri F, Forbes-Hernandez TY, Gasparrini M, Alvarez-Suarez JM, Afrin S, 516 Bompadre S, Quiles JL, Mezzetti B, Battino M. Strawberry as a health promoter: an 517 evidence based review. Food Funct. 2015;6(5):1386-98. DOI:10.1039/c5fo00147a

518 52. Thompson K, Pederick W, Santhakumar AB. Anthocyanins in obesity-associated 519 thrombogenesis: a review of the potential mechanism of action. Food Funct. $520 \quad 2016 ; 7(5): 2169-78$. DOI:10.1039/c6fo00154h

521 53. Weh KM, Aiyer HS, Howell AB, Kresty LA. Cranberry proanthocyanidins modulate 
522 reactive oxygen species in Barrett's and esophageal adenocarcinoma cell lines. J Berry

523 Res. 2016;6(2):125-136. DOI:10.3233/JBR-160122 PMCID:PMC5002987. 


\section{Figure legends}

526 Figure 1. Consort flow diagram of the participants

527 Figure 2. Experimental schedule

528 Figure 3. Changes in cfPWV before and after both NZBC and placebo

$529 *$ : significantly $(\mathrm{P}<0.05)$ different from before.

$530 \uparrow$ : significantly $(\mathrm{P}<0.05)$ different from placebo.

531 cfPWV, carotid-femoral pulse wave velocity; NZBC, New Zealand blackcurrants

532 Figure 4. Changes in central BP before and after both NZBC and placebo

$533 *$ : significantly $(\mathrm{P}<0.05)$ different from before.

$534+\uparrow$ : significantly $(\mathrm{P}<0.01)$ different from placebo.

535 SBP, systolic blood pressure; NZBC, New Zealand blackcurrants 
536 Table 1. Physical characteristics of the participants

$\operatorname{Sex}(\mathrm{F} / \mathrm{M}) \quad 8 / 6$

Age (years)

$73.3 \pm 1.7$

Height (cm)

$159.9 \pm 2.5$

Weight $(\mathrm{kg})$

$55.6 \pm 2.9$

Body mass index $\left(\mathrm{kg} / \mathrm{m}^{2}\right)$

$21.6 \pm 0.7$

Body fat (\%)

$25.7 \pm 1.7$

537 Values are mean \pm SEM. 
538 Table 2. Brachial SBP, DBP, MBP, PP, AIx, and faPWV before and after NZBC and placebo

\begin{tabular}{lcccc}
\hline & \multicolumn{2}{c}{ NZBC } & \multicolumn{2}{c}{ Placebo } \\
& Before & After & Before & After \\
& $142 \pm 5$ & $133 \pm 3^{*}, \dagger \dagger$ & $140 \pm 5$ & $142 \pm 4$ \\
Brachial SBP (mmHg) & $79 \pm 3$ & $76 \pm 2^{*}, \dagger$ & $79 \pm 3$ & $79 \pm 3$ \\
Brachial DBP (mmHg) & $100 \pm 3$ & $95 \pm 3^{* *}, \dagger \dagger$ & $98 \pm 3$ & $100 \pm 3$ \\
Brachial MBP (mmHg) & $63 \pm 4$ & $58 \pm 2 \#, \dagger$ & $60 \pm 3$ & $63 \pm 3$ \\
Brachial PP (mmHg) & & & & \\
AIx (\%) & $87 \pm 4$ & $83 \pm 3^{*}, \dagger$ & $89 \pm 3$ & $86 \pm 3$ \\
faPWV (cm/sec) & $1239 \pm 49$ & $1186 \pm 50$ & $1234 \pm 55$ & $1214 \pm 44$ \\
\hline
\end{tabular}

539 Values are mean \pm SEM. *: significantly $(\mathrm{P}<0.05)$ difference from before. **: significantly $540(\mathrm{P}<0.01)$ difference from before. $\#$ : denotes a strong trend for a different from baseline $(\mathrm{p}=$ 541 0.09). $\dagger$ : significantly $(\mathrm{P}<0.05)$ difference from the placebo. $\dagger \dagger$ : significantly $(\mathrm{P}<0.01)$

542 difference from the placebo. $\$$ : Denotes a strong trend for a different between NZBC and 543 placebo trials $(\mathrm{p}=0.07)$.

544 SBP, systoloc blood pressure; DBP, diastolic blood pressure; MBP, mean blood pressure; PP, 545 pulse pressure; AIx, augmentation index; faPWV, femoral-ankle pulse wave velocity; NZBC, 546 New Zealand blackcurrant 
547 Table 3. Serum Lipids profile and glucose before and after NZBC and placebo

\begin{tabular}{lcccc}
\hline & \multicolumn{2}{c}{ NZBC } & \multicolumn{2}{c}{ Placebo } \\
& Before & After & Before & After \\
& & & & \\
& $88 \pm 12$ & $85 \pm 8$ & $95 \pm 10$ & $93 \pm 10$ \\
TG (mg/dL) & $73 \pm 5$ & $76 \pm 4$ & $71 \pm 6$ & $74 \pm 5$ \\
HDL-C (mg/dL) & $124 \pm 10$ & $120 \pm 8$ & $127 \pm 7$ & $127 \pm 5$ \\
LDL-C (mg/dL) & & & \\
FG (mg/dL) & $91 \pm 4$ & $91 \pm 3$ & $92 \pm 4$ & $91 \pm 4$ \\
\hline
\end{tabular}

548 Values are mean \pm SEM. NZBC, New Zealand blackcurrant; TG, triglycerides; HDL-C, high-

549 density lipoprotein cholesterol; LDL-C, low-density lipoprotein cholesterol; FG, fasting 550 glucose 


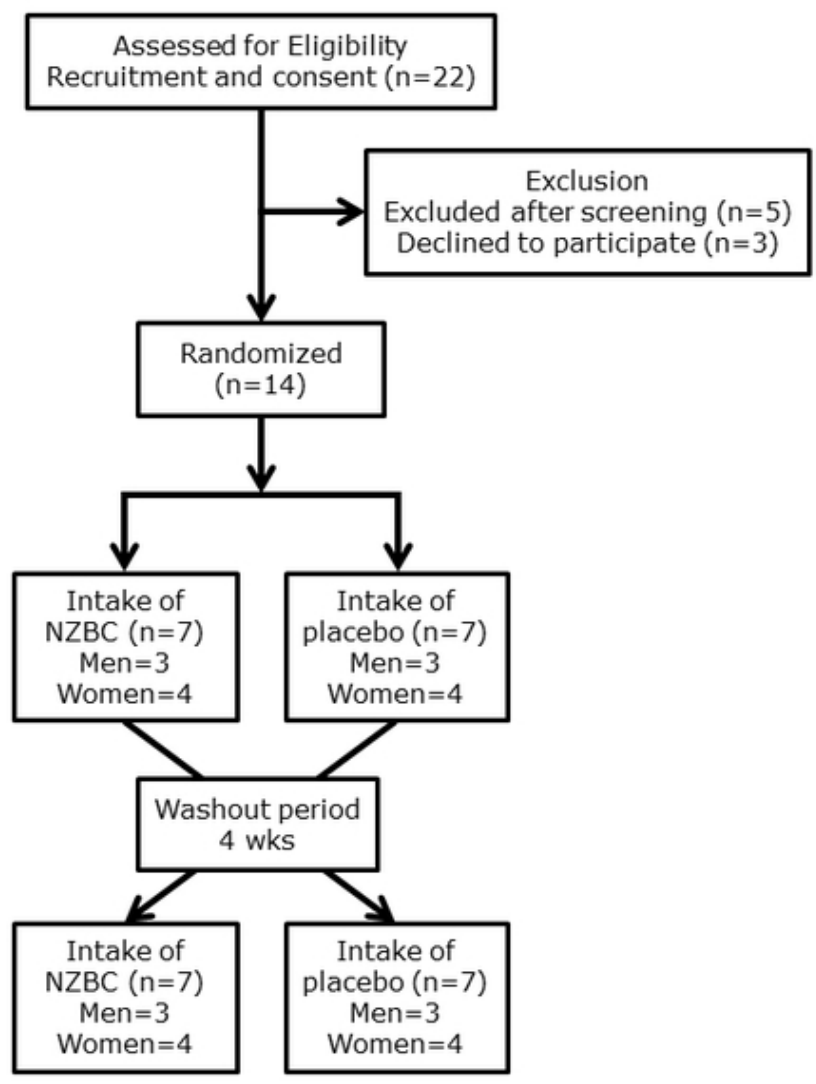

Fig. 1

Figure 1. Consort flow diagram of the participants $52 \times 69 \mathrm{~mm}(300 \times 300 \mathrm{DPI})$ 


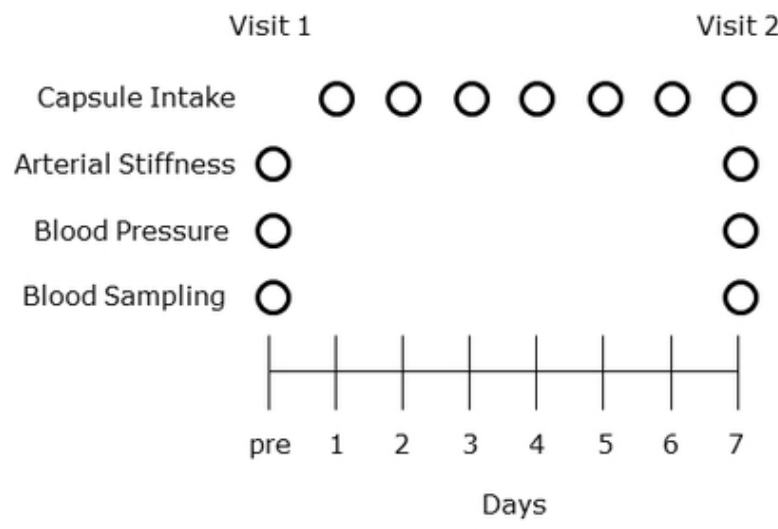

Fig. 2

Figure 2. Experimental schedule

$52 \times 69 \mathrm{~mm}(300 \times 300$ DPI $)$ 


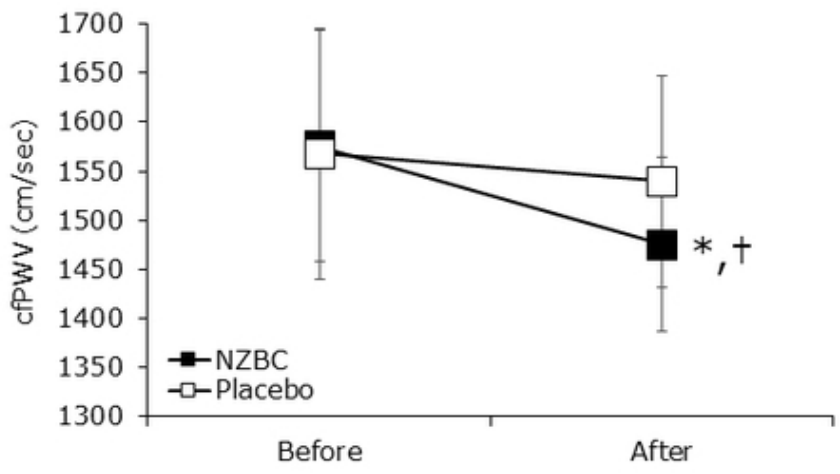

Fig. 3

Figure 3. Changes in cfPWV before and after both NZBC and placebo $52 \times 69 \mathrm{~mm}(300 \times 300 \mathrm{DPI})$ 


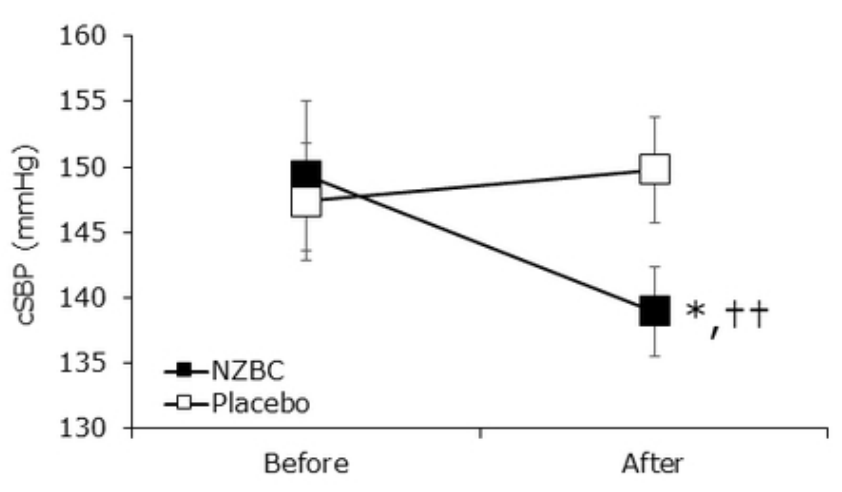

Fig. 4

Figure 4. Changes in central BP before and after both NZBC and placebo $52 \times 69 \mathrm{~mm}(300 \times 300 \mathrm{DPI})$ 